\title{
Genome Sequence of Fusarium oxysporum f. sp. matthiolae, a Brassicaceae Pathogen
}

\author{
Houlin Yu, ${ }^{1,2}$ Dilay Hazal Ayhan, ${ }^{2,3}$ Andrew C. Diener, ${ }^{4, \dagger}$ and Li-Jun Ma ${ }^{1,2,3, \dagger}$ \\ ${ }^{1}$ Plant Biology Graduate Program, University of Massachusetts Amherst, Amherst, MA 01003, U.S.A. \\ ${ }^{2}$ Department of Biochemistry and Molecular Biology, University of Massachusetts Amherst, Amherst, MA \\ 01003, U.S.A. \\ ${ }^{3}$ Molecular and Cellular Biology Graduate Program, University of Massachusetts Amherst, Amherst, MA \\ 01003, U.S.A. \\ ${ }^{4}$ Department of Molecular Biology and Centre for Computational and Integrative Biology, Massachusetts \\ General Hospital, and Department of Genetics, Harvard Medical School, Boston, MA 02114, U.S.A
}

\section{Abstract}

The filamentous fungus Fusarium oxysporum is a soilborne pathogen of many cultivated species and an opportunistic pathogen of humans. F. oxysporum f. sp. matthiolae is one of three formae speciales that are pathogenic to crucifers, including Arabidopsis thaliana, a premier model for plant molecular biology and genetics. Here, we report a genome assembly of $F$. oxysporum f. sp. matthiolae strain PHW726, generated using a combination of PacBio and Illumina sequencing technologies. The genome assembly presented here should facilitate in-depth investigation of $F$. oxysporum-Arabidopsis interactions and shed light on the genetics of fungal pathogenesis and plant immunity.

\section{Genome Announcement}

Strains of the filamentous fungus Fusarium oxysporum, a notorious plant pathogen, can infect hundreds of cultivated species and, at the same time, have distinct host specificity (Kistler 1997; Ma et al. 2013; Michielse and Rep 2009). This host specificity is used to classify F. oxysporum into formae speciales, and a forma specialis typically represents one to a few monophyletic clonal lineages that cause disease in a narrow range of taxonomically related plants (Kistler 1997). At the genomic level, host specificity corresponds to the presence of lineage-specific chromosomes (Ma et al. 2010). However, little is known about molecular mechanisms involved in these host-specific plant-fungus interactions. One of the three formae speciales that are pathogenic to the crucifer Arabidopsis thaliana (Diener and Ausubel 2005; Provart et al. 2016), the genome sequence of $F$. oxysporumf. sp. matthiolae will enable the genetic analysis of fungal pathogenesis and host immunity using the model plant A. thaliana.

Phylogenic analyses indicate that $F$. oxysporum f. sp. matthiolae isolates form a single clonal lineage (Bosland and Williams 1987; Kistler and Benny 1989; Kistler et al. 1987, 1991; O'Donnell et al. 2009), although two races of $F$. oxysporum f. sp. matthiolae are distinguished by the differential susceptibility of varieties of Matthiola incana (Bosland and Williams 1988). Natural variation of immunity is observed among wild accessions or ecotypes of $A$. thaliana toward Fusarium wilt (Diener and Ausubel 2005). Quantitative trait loci mapping in offspring of

${ }^{\dagger}$ Corresponding authors: L.-J. Ma; lijun@ biochem.umass.edu; and

A. Diener; diener@molbio.mgh.harvard.edu

The author(s) declare no conflict of interest.

Accepted for publication 17 January 2020.
Funding

This work was supported by the National Science Foundation (IOS1652641). D. H. Ayhan and L.-J. Ma are also supported by the Burroughs Welcome Foundation (grant BWF1014893) and National Institute of Health (NIH/NEI R01 EY030150).

\section{Keywords}

Arabidopsis thaliana,

Fusarium oxysporum f. sp. matthiolae, genome, microbe-plant molecular interaction, model 
Table 1. Summary of the Fusarium oxysporum f. sp. matthiolae strain PHW726 genome assembly and a comparison with Fol4287 (Ayhan et al. 2018)

\begin{tabular}{lcc} 
& \multicolumn{2}{c}{ Statistics } \\
\cline { 2 - 3 } Variables & PHW726 & Fol4287 \\
Assembly size $(\mathrm{bp})$ & $57,270,650$ & $53,912,367$ \\
Core sequence size (bp) & $43,818,233$ & $42,239,438$ \\
Number of scaffolds & 583 & 499 \\
Number of core scaffolds & 66 & 55 \\
Size of largest scaffold (bp) & $3,557,637$ & $5,733,288$ \\
Interspersed repeat content & $5.35 \%$ & $4.21 \%$ \\
$\mathrm{~N}_{50}$ (bp) & 774,050 & $1,338,693$ \\
$\mathrm{~N}_{90}(\mathrm{bp})$ & 47,752 & 49,310 \\
$\mathrm{~L}_{50}$ (bp) & 18 & 11 \\
$\mathrm{GC}$ content & $47.44 \%$ & $47.68 \%$ \\
\hline
\end{tabular}

crosses between resistant and susceptible ecotypes has identified three RESISTANCE TO F. OXYSPORUM (RFO) genes-one receptor-like protein gene (RFO2) and two receptor-like kinase (RLK) genes (RFO1 and RFO3)-from different RLK gene subfamilies (Cole and Diener 2013; Diener 2013; Diener and Ausubel 2005; Shen and Diener 2013). Because receptor-mediated immunity is reported to be the major determinant of disease resistance to F. oxysporum f. sp. matthiolae (Cole and Diener 2013), investigation of the interaction of F. oxysporumf. sp. matthiolae and $A$. thaliana should lead to a fundamental understanding of receptor-mediated plant immunity, especially against fungal pathogens. The genome sequence described here comes from DNA purified from $F$. oxysporum f. sp. matthiolae race 2, isolated from wilted garden stock ( $M$. incana), a cultivated plant in the crucifer or mustard (Brassicaceae) family, prized for its colorful flowers (Baker 1948; Tatsuzawa et al. 2012). This strain was previously deposited in the American Type Culture Collection (ATCC 16603) by G. M. Armstrong and subsequently designated by P. H. Williams as PHW726 (Kistler et al. 1987).

The pipeline for genome assembly was adapted from Ayhan et al. (2018). Genomic DNA was purified from the mycelium of PHW726, then sequenced by Illumina MiSeq and PacBio RS II platforms with $\times 119$ and $\times 21$ coverage, respectively. We used MiSeq paired-end sequencing with 150 cycles. The maximum size of the PacBio RS II reads was $59 \mathrm{~kb}$ while the mean size was $8.5 \mathrm{~kb}$. Trimmomatic, version 0.32 (Bolger et al. 2014), was used to remove adaptors and trim ends of Illumina reads (parameters: ILLUMINACLIP:TruSeq3-PE.fa:2:30: 10 LEADING:3 TRAILING:3 SLIDINGWINDOW:4:15 MINLEN:36). FastQC, version 0.11.5, was used to check the quality of all reads. SPAdes, version 3.9.1 (Antipov et al. 2016), was used to combine PacBio subreads and trimmed lllumina reads into an initial hybrid assembly with default parameters. BWA, version 0.7.12 (Li and Durbin 2009), was used to map the Illumina reads to the assembly. Further cleaning, fixing, and sorting of mapping reads was done with Picard, version 2.0.1, and Samtools, version 1.3 (Li et al. 2009). A structural variant caller, GRIDSS, version 1.4.1 (Cameron et al. 2017), was used to identify links between scaffolds in the initial assembly. A custom script (scaffolding.m) was used for scaffolding. Minimap2, version 2.17 (Li 2018), was used to map PacBio subreads to new scaffolds, and links were manually inspected and, if necessary, fixed. Further polishing was performed by remapping Illumina reads to the assembly, during which FreeBayes, v0.9.103-g47a713e (Garrison and Marth 2012), was used to identify base variants between reads and the assembly (specially $70 \%$ support of minimal 10 alternate counts, with a minimal base mapping-quality greater than q30). Identified variants were used to correct the assembly by a custom script (FASTAeditWithVCF.m). RepeatMasker 4.0.5 (Tarailo-Graovac and Chen 2009) was used to screen the repeats. Mummer 3.22 (Kurtz et al. 2004) was used to align the assembly with the reference genome assembly for the tomato pathogen F. oxysporum f. sp. lycopersici Fol4287 (Ma et al. 2010).

The final assembly was $57.3 \mathrm{Mb}$ in total length and comprised 583 scaffolds with an $\mathrm{N}_{50}$ value of $0.77 \mathrm{Mb}$ (Table 1). The largest scaffold size was $3.6 \mathrm{Mb}$. The GC content was $47.4 \%$. The size of total interspersed repeats was $3.1 \mathrm{Mb}$, which accounted for $5.4 \%$ of the assembly. A comparison with Fol4287 assembly (Ayhan et al. 2018) suggested a larger assembly size and higher interspersed repeat content of PHW726. The size of sequence 
mapped to the core chromosomes of Fol4287 which, including 66 scaffolds (defined as core scaffolds), was $43.8 \mathrm{Mb}$. The assembly also included a scaffold of $52,365 \mathrm{bp}$ that captured the entire mitochondrial DNA. This Whole Genome Shotgun project has been deposited at DNA Data Bank of Japan/European Nucleotide Archive/GenBank under the accession WJXY00000000. The version described in this article is version WJXY01000000. This assembly for the genome of PHW726 should facilitate future molecular genetics and genomic studies. Candidate F. oxysporum f. sp. matthiolae genes that promote pathogenesis or elicit immune response in $A$. thaliana and $M$. incana can now be predicted, subcloned, and genetically characterized.

\section{Acknowledgments}

We thank R. Ranjan at the University of Massachusetts Amherst Genomic Center and Q. Zeng at the Connecticut Agricultural Experiment Station for their consistent support for our genome projects, and the Massachusetts Green High Performance Computing Center for providing high-performance computing capacity for the genome assembly process.

\section{Author-Recommended Internet Resources}

FastQC: http://www.bioinformatics.babraham.ac.uk/projects/fastqc/

Picard: http://broadinstitute.github.io/picard/

Custom script scaffolding.m: https://github.com/d-ayhan/tools/blob/master/scaffolding.m

Custom script FASTAeditWithVCF.m: https://github.com/d-ayhan/tools/blob/master/FASTAeditWithVCF.m

\section{Literature Cited}

Antipov, D., Korobeynikov, A., McLean, J. S., and Pevzner, P. A. 2016. hybridSPAdes: An algorithm for hybrid assembly of short and long reads. Bioinformatics 32 : 1009-1015.

Ayhan, D. H., López-Díaz, C., Di Pietro, A., and Ma, L.-J. 2018. Improved assembly of reference genome Fusarium oxysporum f. sp. lycopersici Strain Fol4287. Microbiol. Resour. Announce. 7:e00910-18.

Baker, K. F. 1948. Fusarium wilt of garden stock (Mathiola incana). Phytopathology 38:399-403.

Bolger, A. M., Lohse, M., and Usadel, B. 2014. Trimmomatic: A flexible trimmer for Illumina sequence data. Bioinformatics 30:2114-2120.

Bosland, P. W., and Williams, P. H. 1987. An evaluation of Fusarium oxysporum from crucifers based on pathogenicity, isozyme polymorphism, vegetative compatibility, and geographic origin. Can. J. Bot. 65:2067-2073.

Bosland, P. W., and Williams, P. H. 1988. Pathogenicity of geographic isolates of Fusarium oxysporum from crucifers on a differential set of crucifer seedlings. J. Phytopathol. 123:63-68.

Cameron, D. L., Schröder, J., Penington, J. S., Do, H., Molania, R., Dobrovic, A., Speed, T. P., and Papenfuss, A. T. 2017. GRIDSS: Sensitive and specific genomic rearrangement detection using positional de Bruijn graph assembly. Genome Res. 27:2050-2060.

Cole, S. J., and Diener, A. C. 2013. Diversity in receptor-like kinase genes is a major determinant of quantitative resistance to Fusarium oxysporum f. sp. matthioli. New Phytol. 200:172-184.

Diener, A. C. 2013. Routine mapping of Fusarium wilt resistance in $\mathrm{BC}_{1}$ populations of Arabidopsis thaliana. BMC Plant Biol. 13:171.

Diener, A. C., and Ausubel, F. M. 2005. RESISTANCE TO FUSARIUM OXYSPORUM 1, a dominant Arabidopsis disease-resistance gene, is not race specific. Genetics 171:305-321.

Garrison, E., and Marth, G. 2012. Haplotype-based variant detection from short-read sequencing. https://arxiv.org/abs/1207.3907

Kistler, H. C. 1997. Genetic diversity in the plant-pathogenic fungus Fusarium oxysporum. Phytopathology 87:474-479.

Kistler, H. C., and Benny, U. 1989. The mitochondrial genome of Fusarium oxysporum. Plasmid 22:86-89.

Kistler, H. C., Bosland, P. W., Benny, U., Leong, S., and Williams, P. H. 1987. Relatedness of strains of Fusarium oxysporum from crucifers measured by examination of mitochondrial and ribosomal DNA. Phytopathology 77:12891293.
Kistler, H. C., Momol, E. A., and Benny, U. 1991. Repetitive genomic sequences for determining relatedness among strains of Fusarium oxysporum. Phytopathology 81:331-336.

Kurtz, S., Phillippy, A., Delcher, A. L., Smoot, M., Shumway, M., Antonescu, C., and Salzberg, S. L. 2004. Versatile and open software for comparing large genomes. Genome Biol. 5:R12.

Li, H. 2018. Minimap2: Pairwise alignment for nucleotide sequences. Bioinformatics 34:3094-3100

Li, H., and Durbin, R. 2009. Fast and accurate short read alignment with BurrowsWheeler transform. Bioinformatics 25:1754-1760.

Li, H., Handsaker, B., Wysoker, A., Fennell, T., Ruan, J., Homer, N., Marth, G., Abecasis, G., Durbin, R., and 1000 Genome Project Data Processing Subgroup. 2009. The Sequence Alignment/Map format and SAMtools. Bioinformatics 25: 2078-2079.

Ma, L.-J., Geiser, D. M., Proctor, R. H., Rooney, A. P., O'Donnell, K., Trail, F., Gardiner, D. M., Manners, J. M., and Kazan, K. 2013. Fusarium pathogenomics. Annu. Rev. Microbiol. 67:399-416.

Ma, L.-J., van der Does, H. C., Borkovich, K. A., Coleman, J. J., Daboussi, M.-J., Di Pietro, A., Dufresne, M., Freitag, M., Grabherr, M., Henrissat, B., Houterman, P. M., Kang, S., Shim, W.-B., Woloshuk, C., Xie, X., Xu, J.-R., Antoniw, J., Baker, S. E., Bluhm, B. H., Breakspear, A., Brown, D. W., Butchko, R. A. E., Chapman, S., Coulson, R., Coutinho, P. M., Danchin, E. G. J., Diener, A., Gale, L. R., Gardiner, D. M., Goff, S., Hammond-Kosack, K. E., Hilburn, K., Hua-Van, A., Jonkers, W., Kazan, K., Kodira, C. D., Koehrsen, M., Kumar, L., Lee, Y.-H., Li, L., Manners, J. M., Miranda-Saavedra, D., Mukherjee, M., Park, G., Park, J., Park, S.-Y., Proctor, R. H., Regev, A., Ruiz-Roldan, M. C., Sain, D., Sakthikumar, S., Sykes, S., Schwartz, D. C., Turgeon, B. G., Wapinski, I., Yoder, O., Young, S., Zeng, Q., Zhou, S., Galagan, J., Cuomo, C. A., Kistler, H. C., and Rep, M. 2010. Comparative genomics reveals mobile pathogenicity chromosomes in Fusarium. Nature 464:367-373.

Michielse, C. B., and Rep, M. 2009. Pathogen profile update: Fusarium oxysporum. Mol. Plant Pathol. 10:311-324.

O'Donnell, K., Gueidan, C., Sink, S., Johnston, P. R., Crous, P. W., Glenn, A., Riley, R., Zitomer, N. C., Colyer, P., Waalwijk, C., Lee, T., Moretti, A., Kang, S., Kim, H.-S., Geiser, D. M., Juba, J. H., Baayen, R. P., Cromey, M. G., Bithell, S., Sutton, D. A., Skovgaard, K., Ploetz, R., Corby Kistler, H., Elliott, M., Davis, M., and Sarver, B. A. J. 2009. A two-locus DNA sequence database for typing plant and human pathogens within the Fusarium oxysporum species complex. Fungal Genet. Biol. 46:936-948. 
Provart, N. J., Alonso, J., Assmann, S. M., Bergmann, D., Brady, S. M., Brkljacic, J., Browse, J., Chapple, C., Colot, V., Cutler, S., Dangl, J., Ehrhardt, D., Friesner, J. D., Frommer, W. B., Grotewold, E., Meyerowitz, E., Nemhauser, J., Nordborg, M., Pikaard, C., Shanklin, J., Somerville, C., Stitt, M., Torii, K. U., Waese, J., Wagner, D., and McCourt, P. 2016. 50 years of Arabidopsis research: Highlights and future directions. New Phytol. 209:921-944.

Shen, Y., and Diener, A. C. 2013. Arabidopsis thaliana RESISTANCE TO FUSARIUM OXYSPORUM 2 implicates tyrosine-sulfated peptide signaling in susceptibility and resistance to root infection. PLoS Genet. 9: e1003525.

Tarailo-Graovac, M., and Chen, N. 2009. Using RepeatMasker to identify repetitive elements in genomic sequences. Curr. Protoc. Bioinf. 5:4.10.1-4. 10.14.

Tatsuzawa, F., Saito, N., Toki, K., Shinoda, K., and Honda, T. 2012. Flower colors and their anthocyanins in Matthiola incana cultivars (Brassicaceae). J. Jpn. Soc. Hortic. Sci. 81:91-100. 